\title{
DIFFUSIONS IN ONE-DIMENSIONAL BOUNDED DOMAINS WITH REFLECTION, ABSORPTION AND JUMPS AT THE BOUNDARY AND AT SOME INTERIOR POINT
}

\author{
Bohdan Kopytko ${ }^{1}$, Roman Shevchuk ${ }^{2}$ \\ ${ }^{1}$ Institute of Mathematics, Czestochowa University of Technology, Czestochowa, Poland \\ ${ }^{2}$ Ivan Franko National University of Lviv, Lviv, Ukraine \\ 'bohdan.kopytko@im.pcz.pl, ${ }^{2}$ r.v.shevchuk@gmail.com
}

Abstract. By the method of classical potential theory, we obtain the integral representation of the two-parameter operator semigroup that describes the inhomogeneous Feller process on a closed interval $\left[r_{1}, r_{2}\right]$ that is a result of pasting together two diffusion processes given on $\left(r_{1}, r\right)$ and $\left(r, r_{2}\right)$, respectively, where $-\infty<r_{1}<r<r_{2}<\infty$.

\section{Introduction}

Let $C(\bar{D})$ be the Banach space of all real-valued continuous functions on a closed interval $\bar{D}=\left[r_{1}, r_{2}\right]$. Denote by $D_{i}, i=1,2$, the two intervals $\left(r_{1}, r\right)$ and $\left(r, r_{2}\right)$, respectively, where $-\infty<r_{1}<r<r_{2}<\infty$ and by $\varphi_{i}$ the restriction of any function $\varphi$ defined on $\bar{D}$ to the closure $\bar{D}_{i}$.

Assume that the inhomogeneous diffusion process is given on $D_{i}, i=1,2$, and it is generated by the second-order differential operator $A_{s}^{(i)}, s \in[0, T](T>0$ fixed), with the domain of definition $C^{2}\left(\bar{D}_{i}\right)$ :

$$
A_{s}^{(i)} \varphi_{i}(x)=\frac{1}{2} b_{i}(s, x) \frac{d^{2} \varphi_{i}(x)}{d x^{2}}+a_{i}(s, x) \frac{d \varphi_{i}(x)}{d x}, \quad i=1,2,
$$

where the diffusion coefficient $b_{i}(s, x)$ and the drift coefficient $a_{i}(s, x)$ satisfy the conditions:

1) there exist the constants $b$ and $B$ such that $0<b \leq b_{i}(s, x) \leq B$ for all $(s, x) \in[0, T] \times \bar{D}_{i}$

2) for all $s, s^{\prime} \in[0, T], x, x^{\prime} \in \bar{D}_{i}$ the next inequalities hold:

$$
\begin{aligned}
& \left|b_{i}(s, x)-b_{i}\left(s^{\prime}, x^{\prime}\right)\right| \leq c\left(\left|s-s^{\prime}\right|^{\frac{\alpha}{2}}+\left|x-x^{\prime}\right|^{\alpha}\right), \\
& \left|a_{i}(s, x)-a_{i}\left(s^{\prime}, x^{\prime}\right)\right| \leq c\left(\left|s-s^{\prime}\right|^{\frac{\alpha}{2}}+\left|x-x^{\prime}\right|^{\alpha}\right),
\end{aligned}
$$

where $c$ and $\alpha$ are the positive constants, $0<\alpha<1$. 
Define the differential operator $\tilde{A}_{s}, s \in[0, T]$, as follows:

$$
\begin{gathered}
\vartheta\left(\tilde{A}_{s}\right)=\left\{\varphi \in C(\bar{D}): \varphi_{i} \in \vartheta\left(A_{s}^{(i)}\right) \text { for } i=1,2, A_{s}^{(1)} \varphi(r)=A_{s}^{(2)} \varphi(r)\right\}, \\
\tilde{A}_{s} \varphi(x)= \begin{cases}A_{s}^{(1)} \varphi_{1}(x), & x \in \bar{D}_{1}, \\
A_{s}^{(2)} \varphi_{2}(x), & x \in \bar{D}_{2} .\end{cases}
\end{gathered}
$$

Consider also three boundary operators of Feller-Wentzell's type ([1,2]) defined at the points $r, r_{1}, r_{2}$, respectively,

$$
\begin{gathered}
L_{s} \varphi(r)=q_{1}(s) \frac{d \varphi(r-)}{d x}-q_{2}(s) \frac{d \varphi(r+)}{d x}+\gamma(s) \varphi(r)+ \\
+\int_{D_{1} \cup D_{2}}[\varphi(r)-\varphi(y)] \mu(s, d y), \\
L_{s}^{(i)} \varphi\left(r_{i}\right)=(-1)^{i} p_{i}(s) \frac{d \varphi\left(r_{i}\right)}{d x}+\sigma_{i}(s) \varphi\left(r_{i}\right)+\int_{D_{i}}\left[\varphi\left(r_{i}\right)-\varphi(y)\right] \pi_{i}(s, d y),
\end{gathered}
$$

$i=1,2$, where

a) the functions $q_{1}(s), q_{2}(s), \gamma(s), p_{i}(s), \sigma_{i}(s)$ are nonnegative and continuous on $[0, T]$;

b) for a fixed $s, \mu(s, \cdot)$ and $\pi_{i}(s, \cdot)$ are the nonnegative measures on $D_{1} \cup D_{2}$ and $D_{i}$, respectively, such that for all bounded measurable functions $f$ on $\bar{D}$ the integrals

$$
\int_{D_{1} \cup D_{2}}|y-r| f(y) \mu(s, d y), \quad \int_{D_{i}}\left|y-r_{i}\right| f_{i}(y) \pi_{i}(s, d y)
$$

exist and are continuous on $[0, T]$ as functions of $s$;

c) $q_{1}(s)+q_{2}(s)>0$ and $p_{i}(s)>0$ for all $s \in[0, T]$.

It is known (see $[2,3])$ that the boundary conditions

$$
L_{s} \varphi(r)=0, \quad L_{s}^{(i)} \varphi\left(r_{i}\right)=0, \quad i=1,2,
$$

restrict $\tilde{A}_{s}$ to the infinitesimal generator of some Feller semigroup on $\bar{D}$. Such a semigroup is constructed in the present paper. Thus, we are interested in the following problem:

Problem. Construct the two-parameter Feller semigroup $T_{s t}, 0 \leq s<t \leq T$, on $\bar{D}$ whose infinitesimal generator $A_{s}$ is the restriction of $\tilde{A}_{s}$ in (1) to the set of all functions $\varphi \in \vartheta\left(\tilde{A}_{s}\right)$ satisfying the boundary conditions in (2).

This problem is often called the problem of pasting together two one-dimensional diffusion processes or the problem of mathematical modelling of the diffusion phenomenon on $\left[r_{1}, r_{2}\right]$ with three membranes placed at the points 
$r, r_{i}, i=1,2$, respectively (see [4-7]). When the diffusion particle reaches the point $r$ (or $r_{i}, i=1,2$ ), upon the influence of the corresponding membrane, it can be absorbed according to the rates $\gamma$ (or $\sigma_{i}$ ), reflected according to the rates $q_{1}, q_{2}$ (or $p_{i}$ ) and it can jump into the interior according to the rates $\mu$ (or $\pi_{i}$ ).

We investigate the problem using a purely analytic approach and determine the desired semigroup by means of the solution of the corresponding parabolic boundary value problem

$$
\begin{gathered}
\frac{\partial u(s, x, t)}{\partial s}+A_{s}^{(i)} u(s, x, t)=0, \quad 0 \leq s<t \leq T, x \in D_{i}, \quad i=1,2, \\
\lim _{s \uparrow t} u(s, x, t)=\varphi(x), \quad x \in \bar{D}, \\
u(s, r-, t)=u(s, r+, t), \quad 0 \leq s<t \leq T, \\
L_{s} u(s, r, t)=0, \quad 0 \leq s<t \leq T, \\
L_{s}^{(i)} u\left(s, r_{i}, t\right)=0, \quad 0 \leq s<t \leq T, i=1,2 .
\end{gathered}
$$

If $\varphi \in \vartheta\left(A_{s}\right)$, it is clear that the desired function $T_{s t} \varphi$ is to satisfy the equation (3) and the "initial" condition (4). The condition (5) is the consequence of the Feller property of the desired semigroup $T_{s t}$. Since $T_{s t} \varphi \in \vartheta\left(A_{s}\right)$ when $\varphi \in \vartheta\left(A_{s}\right)$, the boundary conditions (6) and (7) are also to be satisfied. Taking into account that the semigroup $T_{s t}$ is to be defined in $C(\bar{D})$, we shall suppose that $\varphi \in C(\bar{D})$.

A classical solvability of the problem (3-7) is established by the boundary integral equations method with the use of the ordinary fundamental solution of equation (3) and associated parabolic potentials. Application of this method permits us not only to prove the existence of the solution of the problem (3)-(7), but to also obtain its integral representation, which is useful in studying the additional properties of the constructed process (see [4]).

Note that in the present paper we generalize the result obtained in [4] where the similar problem was analyzed for the case of two inhomogeneous diffusion processes given in $D_{i}=\left\{x \in \mathbb{R}:(-1)^{i} x>0\right\}, i=1,2$, by the operators $A_{s}^{(i)}$, respectively, with the boundary condition of Feller-Wentzell's type imposed at the origin. We should also mention the works [7-10], where the related problems were studied by the methods of stochastic analysis.

\section{Preliminaries}

Without loss of generality we may suppose that the coefficients $a_{i}(s, x)$ and $b_{i}(s, x)$ in (3) are defined on $[0, T] \times \mathbb{R}$ and the conditions 1), 2) hold for all $(s, x) \in[0, T] \times \mathbb{R}$. We may also suppose that the function $\varphi$ in (4) belongs to $C_{b}(\mathbb{R})$, where $C_{b}(\mathbb{R})$ is the Banach space of real-valued bounded continuous functions on $\mathbb{R}$ with norm 


$$
\|\varphi\|=\sup _{x \in \mathbb{R}}|\varphi(x)| .
$$

Denote by $G_{i}(s, x, t, y), i=1,2$, the fundamental solution of the equation (3) in $[0, T] \times \mathbb{R}$ (its existence is assured by 1$), 2)$ ). Recall that the function $G_{i}$ is nonnegative, continuously differentiable with respect to $s$, twice continuously differentiable with respect to $x$ and can be represented as (see [11])

$$
G_{i}(s, x, t, y)=Z_{i}(s, x, t, y)+Z_{i}^{\prime}(s, x, t, y)
$$

where

$$
Z_{i}(s, x, t, y)=\left[2 \pi b_{i}(t, y)(t-s)\right]^{-\frac{1}{2}} \exp \left\{-\frac{(y-x)^{2}}{2 b_{i}(t, y)(t-s)}\right\},
$$

and the function $Z_{i}^{\prime}$ satisfies the inequality

$$
\left|D_{s}^{r} D_{x}^{p} Z_{i}^{\prime}(s, x, t, y)\right| \leq c(t-s)^{-\frac{1+2 r+p-\alpha}{2}} \exp \left\{-h \frac{(y-x)^{2}}{t-s}\right\}
$$

for all $0 \leq s<t \leq T, x, y \in \mathbb{R}$, where $r$ and $p$ are the nonnegative integers such that $2 r+p \leq 2 ; D_{s}^{r}$ is the partial derivative with respect to $s$ of order $r ; D_{x}^{p}$ is the partial derivative with respect to $x$ of order $p ; c, h$ are positive constants ${ }^{1} ; \alpha$ is the constant in 2). In addition,

$$
\left|D_{s}^{r} D_{x}^{p} G_{i}(s, x, t, y)\right| \leq c(t-s)^{-\frac{1+2 r+p}{2}} \exp \left\{-h \frac{(y-x)^{2}}{t-s}\right\},
$$

where $0 \leq s<t \leq T, x, y \in \mathbb{R}, 2 r+p \leq 2$.

Given the fundamental solution $G_{i}$, we define the parabolic potentials that will be used to solve the problem (3)-(7), namely the Poisson potential

$$
u_{i 0}(s, x, t)=\int_{\mathbb{R}} G_{i}(s, x, t, y) \varphi(y) d y,
$$

and the simple-layer potentials

$$
\begin{aligned}
& u_{i 1}(s, x, t)=\int_{s}^{t} G_{i}(s, x, \tau, r) V_{i}(\tau, t) d \tau, \\
& u_{i 2}(s, x, t)=\int_{s}^{t} G_{i}\left(s, x, \tau, r_{i}\right) V_{i+2}(\tau, t) d \tau,
\end{aligned}
$$

\footnotetext{
${ }^{1}$ We will subsequently denote various positive constants by the same symbol $c$ (or $h$ ).
} 
where $0 \leq s<t \leq T, x \in \bar{D}_{i} ; \varphi$ is the function in (4); $V_{k}, k=\overline{1,4}$, are continuous functions in $s \in[0, t)$ satisfying the inequality

$$
\left|V_{k}(s, t)\right| \leq c(t-s)^{-1+\varepsilon}
$$

for any $\varepsilon>0$.

Note that the functions $u_{i 0}, u_{i 1}, u_{i 2}$ satisfy the equation (3) in the domains $[0, t) \times \bar{D}_{i},[0, t) \times\left(\bar{D}_{i} \backslash\{r\}\right),[0, t) \times\left(\bar{D}_{i} \backslash\left\{r_{i}\right\}\right)$, respectively, and the initial conditions

$$
\begin{gathered}
\lim _{s \uparrow t} u_{i 0}(s, x, t)=\varphi(x), \quad x \in \bar{D}_{i}, \\
\lim _{s \uparrow t} u_{i 1}(s, x, t)=0, \quad x \in \bar{D}_{i} \backslash\{r\}, \quad \lim _{s \uparrow t} u_{i 2}(s, x, t)=0, \quad x \in \bar{D}_{i} \backslash\left\{r_{i}\right\} .
\end{gathered}
$$

In addition, the relations

$$
\begin{gathered}
\left|D_{s}^{r} D_{x}^{p} u_{i 0}(s, x, t)\right| \leq c\|\varphi\|(t-s)^{-\frac{2 r+p}{2}}, 2 r+p \leq 2 \\
\frac{\partial u_{i 1}(s, r \mp, t)}{\partial x}= \pm \frac{V_{i}(s, t)}{b_{i}(s, r)}+\int_{s}^{t} \frac{\partial Z_{i}^{\prime}(s, r, \tau, r)}{\partial x} V_{i}(\tau, t) d \tau \\
\frac{\partial u_{i 2}\left(s, r_{i}, t\right)}{\partial x}=(-1)^{i} \frac{V_{i+2}(s, t)}{b_{i}\left(s, r_{i}\right)}+\int_{s}^{t} \frac{\partial Z_{i}^{\prime}\left(s, r_{i}, \tau, r_{i}\right)}{\partial x} V_{i+2}(\tau, t) d \tau
\end{gathered}
$$

hold.

Note that the last two relations follow from the theorem on the jump of the conormal derivative of a simple-layer potential (see [12, Ch. V, §§2-4]).

\section{Solution of the boundary value problem (3-7)}

The aim of this section is to establish the classical solvability of the boundary value problem (3-7).

We find the solution of (3-7) of the form $\left(x \in \bar{D}_{i}, 0 \leq s<t \leq T\right)$

$$
u(s, x, t)=u_{i 0}(s, x, t)+u_{i 1}(s, x, t)+u_{i 2}(s, x, t)
$$

with the unknown functions $V_{k}, k=\overline{1,4}$, to be determined. Substituting (14) into boundary conditions (5-7), we get, upon using the relations $(12,13)$, the following system of integral equations for $V_{i}$ :

$$
\begin{gathered}
\sum_{i=1}^{2} \int_{s}^{t}(-1)^{i}\left[G_{i}(s, r, \tau, r) V_{i}(\tau, t)+G_{i}\left(s, r, \tau, r_{i}\right) V_{i+2}(\tau, t)\right] d \tau=\Delta(s, t) \\
0 \leq s<t \leq T
\end{gathered}
$$




$$
\begin{gathered}
\sum_{i=1}^{2}\left(\frac{q_{i}(s) V_{i}(s, t)}{b_{i}(s, r)}+\int_{s}^{t}\left[R_{i}(s, \tau) V_{i}(\tau, t)+R_{i+2}(s, \tau) V_{i+2}(\tau, t)\right] d \tau\right) \\
=\Lambda(s, t), \quad 0 \leq s<t \leq T \\
\frac{p_{i}(s) V_{i+2}(s, t)}{b_{i}\left(s, r_{i}\right)}+\int_{s}^{t}\left[N_{i}(s, \tau) V_{i}(\tau, t)+N_{i+2}(s, \tau) V_{i+2}(\tau, t)\right] d \tau \\
=\Upsilon_{i}(s, t), \quad 0 \leq s<t \leq T, \quad i=1,2
\end{gathered}
$$

where

$$
\begin{gathered}
\Delta(s, t)=u_{10}(s, r, t)-u_{20}(s, r, t), \quad \Lambda(s, t)=-\sum_{i=1}^{2} P_{s}^{(i)} u_{i 0}(s, r, t), \\
\Upsilon_{i}(s, t)=-L_{s}^{(i)} u_{i 0}\left(s, r_{i}, t\right), \\
R_{i}(s, \tau)=\left.P_{s}^{(i)} G_{i}(s, x, \tau, r)\right|_{x=r}, \quad R_{i+2}(s, \tau)=\left.P_{s}^{(i)} G_{i}\left(s, x, \tau, r_{i}\right)\right|_{x=r}, \\
N_{i}(s, \tau)=\left.L_{s}^{(i)} G_{i}(s, x, \tau, r)\right|_{x=r_{i}}, \quad N_{i+2}(s, \tau)=\left.L_{s}^{(i)} G_{i}\left(s, x, \tau, r_{i}\right)\right|_{x=r_{i},} \\
P_{s}^{(i)} f(x)=(-1)^{i+1} q_{i}(s) \frac{d f(x)}{d x}+\frac{\gamma(s)}{2} f(x)+\int_{D_{i}}[f(x)-f(y)] \mu(s, d y) .
\end{gathered}
$$

Note that equation (15) is the Volterra integral equation of the first kind and equation (16), as well as two equations in (17), are the ones of the second kind. Applying the Holmgren transform

$$
\mathcal{E}_{s t} \Delta(s, t)=\sqrt{\frac{2}{\pi}} \frac{d}{d s} \int_{s}^{t}(\rho-s)^{-\frac{1}{2}} \Delta(\rho, t) d \rho, \quad 0 \leq s<t \leq T,
$$

to both sides of (15), after some straightforward simplifications, we obtain the equivalent Volterra integral equation of the second kind

$$
\begin{gathered}
\sum_{i=1}^{2}(-1)^{i+1}\left(\frac{V_{i}(s, t)}{\sqrt{b_{i}(s, r)}}-\int_{s}^{t}\left[Q_{i}(s, \tau) V_{i}(\tau, t)++Q_{i+2}(s, \tau) V_{i+2}(\tau, t)\right] d \tau\right) \\
=\Phi(s, t)
\end{gathered}
$$

where

$$
\begin{gathered}
\Phi(s, t)=\frac{1}{\sqrt{2 \pi}} \int_{s}^{t}(\rho-s)^{-\frac{3}{2}}(\Delta(\rho, t)-\Delta(s, t)) d \rho-\sqrt{\frac{2}{\pi}} \Delta(s, t)(t-s)^{-\frac{1}{2}}, \\
Q_{i}(s, \tau)=\varepsilon_{s \tau} Z_{i}^{\prime}(s, r, \tau, r), Q_{i+2}(s, \tau)=\mathcal{E}_{s \tau} G_{i}\left(s, r, \tau, r_{i}\right) .
\end{gathered}
$$


Next, by means of elementary transformations we reduce the system (18), (16), (17) to

$$
V_{i}(s, t)=\sum_{j=1}^{4} \int_{s}^{t} K_{i j}(s, \tau) V_{j}(\tau, t)+\Psi_{i}(s, t), \quad i=\overline{1,4}
$$

where

$$
\begin{aligned}
& \Psi_{i}(s, t)= \begin{cases}d_{i}(s)\left(\Lambda(s, t)+(-1)^{i+1} \frac{q_{3-i}}{\sqrt{b_{3-i}}} \Phi(s, t)\right), & i=1,2, \\
d_{i}(s) \Upsilon_{i-2}(s, t), & i=3,4\end{cases} \\
& K_{i j}(s, \tau)=\left\{\begin{array}{l}
d_{i}(s)\left(-R_{j}(s, \tau)+(-1)^{i+j} \frac{q_{3-i}(s)}{\sqrt{b_{3-i}(s, r)}} Q_{j}(s, \tau)\right), \quad i=1,2, \\
-d_{i}(s) N_{j}(s, \tau), \quad(i=j \quad \text { or } i-2=j) \quad \text { and } i=3,4 \\
0, \quad \text { otherwise. }
\end{array}\right. \\
& d_{i}(s)= \begin{cases}\frac{b_{i}(s, r) \sqrt{b_{3-i}(s, r)}}{q_{1} \sqrt{b_{2}(s, r)}+q_{2} \sqrt{b_{1}(s, r)}}, & i=1,2, \\
\frac{b_{i-2}\left(s, r_{i-2}\right)}{p_{i-2}(s)}, & i=3,4 .\end{cases}
\end{aligned}
$$

Let us estimate the functions $\Psi_{i}$ in (19). For this purpose, we consider the integrals

$$
\begin{gathered}
I_{i}(s, t)=\int_{D_{i}}\left[u_{i 0}\left(s, r_{i}, t\right)-u_{i 0}(s, y, t)\right] \pi_{i}(s, d y), \quad i=1,2, \\
J(s, t)=\int_{s}^{t}(\rho-s)^{-\frac{3}{2}}(\Delta(\rho, t)-\Delta(s, t)) d \rho
\end{gathered}
$$

which are the terms in the expression for $\Psi_{i}$. Applying the Lagrange formula to the integrand $u_{i 0}\left(s, r_{i}, t\right)-u_{i 0}(s, y, t)$ we obtain

$$
I_{i}(s, t)=\left.\int_{D_{i}}\left(y-r_{i}\right) \frac{\partial u_{i 0}(s, x, t)}{\partial x}\right|_{x=r_{i}+\theta\left(y-r_{i}\right)} \pi_{i}(s, d y), \quad 0<\theta<1 .
$$

Then using the inequality (11) when $r=0$ and $p=1$, we find that

$$
\left|I_{i}(s, t)\right| \leq c\|\varphi\|(t-s)^{-\frac{1}{2}}
$$


To estimate the function $J(s, t)$ we write it in the form

$$
\begin{gathered}
J(s, t)=\int_{s}^{\frac{s+t}{2}}(\rho-s)^{-\frac{3}{2}}(\Delta(\rho, t)-\Delta(s, t)) d \rho \\
+\int_{\frac{s+t}{2}}^{t}(\rho-s)^{-\frac{3}{2}}(\Delta(\rho, t)-\Delta(s, t)) d \rho .
\end{gathered}
$$

Denote the first term in (20) by $J_{1}(s, t)$ and the second one by $J_{2}(s, t)$. If we apply the Lagrange formula to the increments $\Delta(\rho, t)-\Delta(s, t)$ in the expression for $J_{1}$ and then use the inequality (11) (when $r=1, p=0$ ), we get

$$
\begin{aligned}
& \left|J_{1}(s, t)\right| \leq\left|\int_{s}^{\frac{s+t}{2}}(\rho-s)^{-\frac{1}{2}} \cdot \frac{\partial \Delta\left(s_{0}, t\right)}{\partial s}\right|_{s_{0}=s+\theta(\rho-s)} d \rho \mid \\
& \leq c\|\varphi\| \int_{s}^{\frac{s+t}{2}}(\rho-s)^{-\frac{1}{2}}[t-s-\theta(\rho-s)] \leq c\|\varphi\|(t-s)^{-\frac{1}{2}} .
\end{aligned}
$$

For $J_{2}(s, t)$ we have

$$
\left|J_{2}(s, t)\right| \leq\left(\frac{t-s}{2}\right)^{-\frac{3}{2}} \int_{\frac{s+t}{2}}^{t}(|\Delta(\rho, t)|+|\Delta(s, t)|) d \rho \leq c\|\varphi\|(t-s)^{-\frac{1}{2}}
$$

Estimating all the rest of the terms in formula for $\Psi_{i}$, we conclude that

$$
\left|\Psi_{i}(s, t)\right| \leq c_{0}\|\varphi\|(t-s)^{-\frac{1}{2}}
$$

where $c_{0}$ is some positive constant.

Proceeding by the same considerations as the ones leading to the estimation (21) we can also investigate the kernels $K_{i j}$ in (19). We obtain the following result: the kernels $K_{i j}(s, \tau), 0 \leq s<\tau<t \leq T$, can be represented as

$$
K_{i j}(s, \tau)=H_{i j}(s, \tau)+H_{i j}^{\prime}(s, \tau), \quad i, j=\overline{1,4}
$$

where $H_{i j}(s, \tau)=$ 


$$
=\left\{\begin{array}{l}
d_{i}(s) \int_{D_{j}^{\delta}(r)}\left[Z_{j}(s, y, \tau, r)-Z_{j}(s, r, \tau, r)\right] \mu(s, d y), \quad i, j=1,2, \\
d_{i}(s) \int_{\substack{D_{j-2}^{\delta}\left(r_{j-2}\right) \\
0,}}\left[Z_{j-2}\left(s, y, \tau, r_{j-2}\right)-Z_{j-2}\left(s, r_{j-2}, \tau, r_{j-2}\right)\right] \pi_{j-2}(s, d y), \quad i=j=3,4, \\
0 \text { otherwise, }
\end{array}\right.
$$

and $H_{i j}^{\prime}(s, \tau)$ satisfy the inequality

$$
\left|H_{i j}^{\prime}(s, \tau)\right| \leq h(\delta)(\tau-s)^{-1+\frac{\alpha}{2}}
$$

Here $\delta, h(\delta)$ are any positive number and some constant depending on $\delta$, respectively; $D_{j}^{\delta}(x)=\left\{y \in D_{j}: \quad|y-x|<\delta\right\}$. Note also that

$$
H_{i j}^{\prime}(s, \tau) \equiv 0 \quad \text { when } \quad i \in\{3,4\} \cap\{i \neq j \text { and } i-2 \neq j\} .
$$

Thus kernels $K_{i j}$ have non-integrable singularity, which is caused by $H_{i j}$, and therefore we do not know yet whether a solution of (19) exists. We shall see presently that it is nevertheless possible to obtain the solution of (19) by the method of successive approximations, i.e.,

$$
V_{i}(s, t)=\sum_{k=0}^{\infty} V_{i}^{(k)}(s, t), \quad 0 \leq s<t \leq T, \quad i=\overline{1,4}
$$

where

$$
\begin{gathered}
V_{i}^{(0)}(s, t)=\Psi_{i}(s, t) \\
V_{i}^{(k)}(s, t)=\sum_{j=1}^{4} \int_{s}^{t} K_{i j}(s, \tau) V_{j}^{(k-1)}(\tau, t) d \tau, \quad k=1,2, \ldots
\end{gathered}
$$

Let us estimate the function $V_{i}^{(1)}$. We can write

$$
\begin{gathered}
V_{i}^{(1)}(s, t)=\sum_{j=1}^{4} \int_{s}^{t} K_{i j}(s, \tau) V_{j}^{(0)}(\tau, t) d \tau=\sum_{j=1}^{4} \int_{s}^{t} H_{i j}(s, \tau) \Psi_{j}(\tau, t) d \tau+ \\
\quad+\sum_{j=1}^{4} \int_{s}^{t} H_{i j}^{\prime}(s, \tau) \Psi_{j}(\tau, t) d \tau=\tilde{V}_{i}^{(1)}(s, t)+\widehat{V}_{i}^{(1)}(s, t)= \\
=\left\{\begin{array}{l}
\sum_{j=1}^{2} \int_{s}^{t} H_{i j}(s, \tau) \Psi_{j}(\tau, t) d \tau+\sum_{j=1}^{4} \int_{s}^{t} H_{i j}^{\prime}(s, \tau) \Psi_{j}(\tau, t) d \tau, \quad i=1,2, \\
\int_{s}^{t} H_{i i}(s, \tau) \Psi_{i}(\tau, t) d \tau+\sum_{j \in\{i, i-2\}} \int_{s}^{t} H_{i j}^{\prime}(s, \tau) \Psi_{j}(\tau, t) d \tau, \quad i=3,4 .
\end{array}\right.
\end{gathered}
$$


From (21) and (23) it follows that

$$
\left|\tilde{V}_{i}^{(1)}(s, t)\right| \leq 4 c_{0} h(\delta)\|\varphi\| \frac{\Gamma\left(\frac{\alpha}{2}\right) \Gamma\left(\frac{1}{2}\right)}{\Gamma\left(\frac{1+\alpha}{2}\right)}(t-s)^{-\frac{1-\alpha}{2}}, \quad i=\overline{1,4}
$$

To show how we estimate $\tilde{V}_{i}^{(1)}$ it suffices to consider any of the integrals $\int_{S}^{t} H_{i j} \Psi_{j} d \tau$. Let us take $i, j=1,2$ and denote these integrals by $\tilde{I}_{i j}(s, t)$. Then

$$
\begin{gathered}
\left|\tilde{I}_{i j}(s, t)\right| \leq \frac{c_{0}\|\varphi\| d_{i}(s)}{\sqrt{2 \pi b}}\left|\int_{s}^{t}(t-\tau)^{-\frac{1}{2}}(\tau-s)^{-\frac{1}{2}} d \tau \int_{D_{j}^{\delta}(r)}\left(e^{\frac{-(y-r)^{2}}{2 b \cdot(\tau-s)}}-1\right) \mu(s, d y)\right|= \\
=\frac{c_{0}\|\varphi\| d_{i}(s)}{\sqrt{2 \pi b}}\left|\int_{S}^{t}(t-\tau)^{-\frac{1}{2}}(\tau-s)^{-\frac{1}{2}} d \tau \int_{D_{j}^{\delta}(r)} \mu(s, d y) \int_{0}^{1} \frac{\partial}{\partial \theta} e^{\frac{-\theta(y-r)^{2}}{2 b \cdot(\tau-s)}} d \theta\right|= \\
=\frac{c_{0}\|\varphi\| d_{i}(s)}{2 b \sqrt{2 \pi b}}\left|\int_{D_{j}^{\delta}(r)}(y-r) \mu(s, d y) \int_{0}^{1}(y-r) e^{\frac{-\theta(y-r)^{2}}{2 b \cdot(t-s)}} d \theta \int_{S}^{t} \frac{e^{\frac{-\theta(y-r)^{2}}{2 b \cdot(t-s)} \cdot \frac{t-\tau}{\tau-s}}}{(t-\tau)^{\frac{1}{2}}(\tau-s)^{\frac{3}{2}}} d \tau\right|,
\end{gathered}
$$

where $b$ is the constant in 1). The change of variables $z=\frac{t-\tau}{\tau-s}$ in the inner integral in the last relation leads to

$$
\begin{gathered}
\left|\tilde{I}_{i j}\right| \leq \frac{c_{0}\|\varphi\| d_{i}(s)}{2 b \sqrt{2 \pi b}(t-s)}\left|\int_{D_{j}^{\delta}(r)}(y-r) \mu(s, d y) \int_{0}^{1}(y-r) e^{\frac{-\theta(y-r)^{2}}{2 b \cdot(t-s)}} d \theta \int_{0}^{\infty} z^{-\frac{1}{2}} e^{\frac{-\theta(y-r)^{2}}{2 b \cdot(t-s)} \cdot z} d z\right|= \\
\quad \leq c_{0}\|\varphi\|(t-s)^{-\frac{1}{2} \frac{d_{i}(s)}{b}} \int_{D_{j}^{\delta}(r)}|y-r| \mu(s, d y) .
\end{gathered}
$$

It is obvious that the same estimate holds for $i=3,4$ (with integral term $\left.\int_{D_{i-2}^{\delta}\left(r_{i-2}\right)}\left|y-r_{i-2}\right| \pi_{i-2}(s, d y)\right)$.

Introducing the following notations:

$$
\begin{gathered}
d=\max _{s \in[0, T]}\left\{d_{i}(s): \quad i=\overline{1,4}\right\}, \\
m(\delta)=\frac{d}{b} \max _{s \in[0, T]}\left\{\sum_{j=1}^{2} \int_{D_{j}^{\delta}(r)}|y-r| \mu(s, d y), \quad \int_{D_{i}^{\delta}\left(r_{i}\right)}\left|y-r_{i}\right| \pi_{i}(s, d y), \quad i=1,2\right\},
\end{gathered}
$$

we derive the estimate

$$
\left|\tilde{V}_{i}^{(1)}(s, t)\right| \leq c_{0} m(\delta)\|\varphi\|(t-s)^{-\frac{1}{2}}, \quad i=\overline{1,4} .
$$


Combining (25) and (26), we conclude that

$$
\left|V_{i}^{(1)}(s, t)\right| \leq c_{0}\|\varphi\|(t-s)^{-\frac{1}{2}}\left(\frac{4 h(\delta) T^{\frac{\alpha}{2}} \Gamma\left(\frac{\alpha}{2}\right) \cdot \Gamma\left(\frac{1}{2}\right)}{\Gamma\left(\frac{1+\alpha}{2}\right)}+m(\delta)\right),
$$

By means of the method of mathematical induction, we prove that the terms $V_{i}^{(k)}$ of series (24) satisfy the inequalities

$$
\left|V_{i}^{(k)}(s, t)\right| \leq c\|\varphi\|(t-s)^{-\frac{1}{2}} \sum_{n=0}^{k} C_{k}^{n} \cdot a^{(k-n)} m(\delta)^{n}, \quad k=0,1,2, \ldots
$$

where

$$
a^{(n)}=\frac{\left(4 h(\delta) T^{\frac{\alpha}{2}} \Gamma\left(\frac{\alpha}{2}\right)\right)^{n} \cdot \Gamma\left(\frac{1}{2}\right)}{\Gamma\left(\frac{1+n \alpha}{2}\right)}, \quad n=0,1,2, \ldots, k .
$$

Fix $\delta=\delta_{0}$ such that $m\left(\delta_{0}\right)<1$. Then in view of (28), we establish that

$$
\sum_{k=0}^{\infty}\left|V_{i}^{(k)}(s, t)\right| \leq c\|\varphi\|(t-s)^{-\frac{1}{2}} \sum_{k=0}^{\infty} \frac{\left(\frac{4 h\left(\delta_{0}\right)}{1-m\left(\delta_{0}\right)} T^{\frac{\alpha}{2}} \Gamma\left(\frac{\alpha}{2}\right)\right)^{k}}{\Gamma\left(\frac{1+k \alpha}{2}\right)} \cdot \frac{\Gamma\left(\frac{1}{2}\right)}{1-m\left(\delta_{0}\right)} .
$$

Hence, the series (24) converges absolutely in $0 \leq s<t \leq T$. This implies that functions $V_{i}, i=\overline{1,4}$, exist and satisfy the inequality

$$
\left|V_{i}(s, t)\right| \leq c\|\varphi\|(t-s)^{-\frac{1}{2}}, \quad 0 \leq s<t \leq T .
$$

We have thus constructed a solution $u(s, x, t)$ of the problem (3-7) which is of the form (14). Using the relations (8-11) and the estimate (29) it is easy to verify that

$$
u(s, x, t) \in C^{1,2}\left([0, t) \times D_{1} \cup D_{2}\right) \cap C([0, t] \times \bar{D}) .
$$

Concerning the uniqueness of the solution of (3-7), note that it follows from the maximum principle (see [12, Ch. II]).

We have proved the following theorem:

Theorem 1. Let the conditions 1), 2) and a)-c) hold, and let $\varphi \in C(\bar{D})$. Then the problem (3-7) has a unique solution

$$
u(s, x, t) \in C^{1,2}\left([0, t) \times D_{1} \cup D_{2}\right) \cap C([0, t] \times \bar{D}) .
$$


If the coefficients of operators $A_{s}^{(i)}$ are extended to $[0, T] \times \mathbb{R}$ such that properties $1)$, 2) hold for all $(s, x) \in[0, T] \times \mathbb{R}$ and the function $\varphi$ is extended to $\mathbb{R}$ such that $\varphi \in C_{b}(\mathbb{R})$, then the solution of (3-7) can be represented as

$$
\begin{gathered}
u(s, x, t)=\int_{\mathbb{R}} G_{i}(s, x, t, y) \varphi(y) d y+\int_{s}^{t} G_{i}(s, x, \tau, r) V_{i}(\tau, t)+ \\
+\int_{s}^{t} G_{i}\left(s, x, \tau, r_{i}\right) V_{i+2}(\tau, t) d \tau, \quad 0 \leq s \leq t \leq T, \quad x \in \bar{D}_{i}, \quad i=1,2,
\end{gathered}
$$

where $G_{i}$ is the fundamental solution of $\frac{\partial}{\partial s}+A_{s}^{(i)}$ and the collection $\left(V_{k}\right)_{k=\overline{1,4}}$ is the solution of the system of Volterra integral equations of the second kind (19).

Remark. The assertion of uniqueness also implies that the solution $u(s, x, t), 0 \leq s \leq t \leq T, x \in \bar{D}$, of problems (3-7) does not depend on which extensions of functions $a_{i}(s, x), b_{i}(s, x)$ and $\varphi(x)$ are chosen.

\section{Diffusion phenomenon on $\left[r_{1}, r_{2}\right]$}

We introduce the two-parameter family of linear operators

$$
T_{s t} \varphi(x)=u(s, x, t, \varphi), \quad 0 \leq s \leq t \leq T, \quad x \in \bar{D}, \quad \varphi \in C_{b}(\mathbb{R}),
$$

where $u(s, x, t, \varphi)$ is the solution of the problem (3)-(7) with function $\varphi$ in (4), and proceeds to study its properties in the space $C_{b}(\mathbb{R})$.

First we note that

$$
\lim _{n \rightarrow \infty} T_{s t} \varphi_{n}(x)=T_{s t} \varphi(x), \quad 0 \leq s \leq t \leq T, \quad x \in \bar{D},
$$

for every sequence of functions $\varphi_{n} \in C_{b}(\mathbb{R})$ such that

$$
\sup _{n}\left\|\varphi_{n}\right\|<\infty \quad \text { and } \quad \lim _{n \rightarrow \infty} \varphi_{n}(x)=\varphi(x), \quad x \in \bar{D} .
$$

This property easily follows from Lebesgue bounded convergence theorem.

We next prove that the operators $T_{s t}, 0 \leq s \leq t \leq T$, are positivity preserving.

Lemma. If $\varphi \in C_{b}(\mathbb{R})$ and $\varphi(x) \geq 0$ for all $x \in \bar{D}$, then $T_{s t} \varphi(x) \geq 0$ for all $0 \leq s \leq t \leq T, x \in \bar{D}$.

Suppose that $T_{s t} \varphi(x)$ takes negative values in $[0, t] \times \bar{D}$ and denote by $m_{*}$ its minimum in $[0, t] \times \bar{D}$. Then, by the minimum principle, value $m_{*}$ is attained at some point $\left(s_{0}, x_{0}\right) \in(0, t) \times\left\{r_{1}, r, r_{2}\right\}$. In case $x_{0}=r$ (i.e., $\left.T_{s_{0} t} \varphi(r)=m_{*}<0\right)$, the inequalities 


$$
\gamma\left(s_{0}\right) T_{s_{0} t} \varphi(r) \leq 0, \quad \int_{D_{1} \cup D_{2}}\left[T_{s_{0} t} \varphi(r)-T_{s_{0} t} \varphi(y)\right] \mu(s, d y) \leq 0
$$

hold. Furthermore, Theorem 14 in [12, p. 69] implies that

$$
\frac{\partial T_{s_{0} t} \varphi(r-)}{\partial x}<0, \quad \frac{\partial T_{s_{0} t} \varphi(r+)}{\partial x}>0 .
$$

But since $q_{1}\left(s_{0}\right)+q_{2}\left(s_{0}\right)>0$, we have $L_{s_{0}} T_{S_{0} t} \varphi(r)<0$. This contradicts (6). Similarly, the case $x_{0}=r_{i}, i \in\{1,2\}$ leads us to the inequality $L_{s_{0}} T_{S_{0} t} \varphi\left(r_{i}\right)<0$ which contradicts (7). A contradiction we arrived at indicates that $m_{*} \geq 0$. This completes the proof of the lemma.

By similar considerations to those in proof of Lemma 1, one can easily verify that the operators $T_{s t}$ are contractive, i.e.,

$$
\left\|T_{s t}\right\| \leq 1, \quad 0 \leq s \leq t \leq T .
$$

Note also that the operator family $T_{s t}$ has a semigroup property

$$
T_{s t}=T_{s \tau} T_{\tau t}, \quad 0 \leq s \leq \tau \leq t \leq T,
$$

This property is a consequence of the assertion of uniqueness of the solution of the problem (3-7). Indeed, considering the problem (3-7) in the time interval $[s, \tau]$ with the function $T_{\tau t} \varphi, \tau \leq t \leq T$, taken as the "initial" function, we deduce that $T_{s \tau}\left(T_{\tau t} \varphi\right), 0 \leq s \leq \tau \leq t \leq T$, is the solution of (3-7) with the function $\varphi$ in (4), and hence (31) follows.

These properties allow us to assert (see [13], Ch. II) that $T_{s t}, 0 \leq s \leq t \leq T$, is a Feller semigroup on $\bar{D}$ for which there exists a unique transition function $P(s, x, t, \cdot)$ on $\bar{D}$ such that

$$
T_{s t} \varphi(x)=\int_{\bar{D}} P(s, x, t, d y) \varphi(y), \quad 0 \leq s \leq t \leq T, \quad x \in \bar{D}, \quad \varphi \in C_{b}(\mathbb{R}) .
$$

Thus, we have proved the following theorem:

Theorem 2. Let the conditions of Theorem 1 hold. Then the two-parameter semigroup of operators $T_{s t}, 0 \leq s \leq t \leq T$, defined by formula (30) describes the inhomogeneous Feller process on $\bar{D}$ such that on $D_{1}$ and $D_{2}$ it coincides with the diffusion processes generated by $A_{s}^{(1)}$ and $A_{s}^{(2)}$, respectively, and its behavior at each of points $r, r_{i}, i=1,2$, is determined by the corresponding boundary condition in (2). 


\section{References}

[1] Feller W., Diffusion processes in one dimension, Trans. Amer. Math. Soc. 1954, 77, 1-31.

[2] Wentzell A.D., Semigroups of operators that correspond to a generalized differential operator of second order, Dokl. AN SSSR 1956, 111, 2, 269-272 (in Russian).

[3] Langer H., Schenk W., Knotting of one-dimensional Feller process, Math. Nachr. 1983, 113, 151-161.

[4] Kopytko B.I., Shevchuk R.V., On pasting together two inhomogeneous diffusion processes on a line with the general Feller-Wentzell conjugation condition, Theory of Stochastic Processes 2011, 17(33), 2, 55-70.

[5] Shevchuk R.V., Pasting of two one-dimensional diffusion processes, Annales Mathematicae et Informaticae 2012, 39, 225-239.

[6] Kononchuk P.P., Kopytko B.I., Semigroups of operators that describe a Feller process on the line, which is the result of pasting together two diffusion processes, Theor. Probability and Math. Statist. 2012, 84, 87-97.

[7] Portenko N.I., Generalized Diffusion Processes, Naukova Dumka, Kyiv 1982 (English transl., American Mathematical Society, Providence, RI 1990).

[8] Anulova S.V., On stochastic differential equations with boundary conditions in a half-plane, Izv. AN SSSR Ser. Mat. 1981, 45, 3, 491-508 (in Russian).

[9] Pilipenko A.Yu., On the Skorokhod mapping for equations with reflection and possible jumplike exit from a boundary, Ukrainian Math. J. 2012, 63, 9, 1415-1432.

[10] Kulinic G.L., On the limit behavior of the distribution of the solution of a stochastic diffusion equation, Theory Probability Appl. 1967, 12, 3, 497-499.

[11] Ladyzhenskaya O.A., Solonnikov V.A., Ural'tseva N.N., Linear and Quasilinear Equations of Parabolic Type, Nauka, Moscow 1967 (in Russian).

[12] Friedman A., Partial Differential Equations of Parabolic Type, Mir, Moscow 1968 (in Russian).

[13] Dynkin E.B., Markov Processes, Fizmatgiz, Moscow 1963 (in Russian). 